01

\title{
Расчет термического сопротивления теплораспределителя системы охлаждения теплонагруженного элемента
}

\author{
(C) Е.Н. Васильев
}

Институт вычислительного моделирования СО РАН, 660036 Красноярск, Россия

e-mail: ven@icm.krasn.ru

(Поступило в Редакцию 26 апреля 2017 г.)

\begin{abstract}
Проведено численное моделирование теплообмена в теплораспределителе термоэлектрической системы охлаждения, размещенном между теплонагруженным элементом и термоэлектрическим модулем для согласования их размеров и выравнивания теплового потока. Получены зависимости характерных значений температуры и термического сопротивления теплораспределителя из меди и алюминия от его толщины и размера теплонагруженного элемента. Проведен сравнительный анализ влияния теплопроводности материала и геометрических параметров на величину термического сопротивления. Определена оптимальная толщина теплораспределителя в зависимости от размера теплонагруженного элемента.
\end{abstract}

DOI: 10.21883/JTF.2018.04.45714.2312

\section{Введение}

Миниатюризация электронных и технических компонентов, сочетающаяся с ростом мощности энергопотребления, обостряет проблему обеспечения оптимальных температурных режимов. Перегрев теплонагруженных элементов (ТНЭ) может приводить к негативному изменению рабочих характеристик, снижению надежности и ресурса, выходу из строя. Установлено, что при изменении температуры радиоэлектронных блоков от 40 до $70^{\circ} \mathrm{C}$ интенсивность отказов увеличивается в 2-6 раз [1]. Применение термоэлектрических модулей (ТЭМ) значительно расширяет возможности охлаждения и терморегулирования ТНЭ. В то же время эффективность термоэлектрических систем охлаждения и терморегулирования (ТЭСОТ) сравнительно невысока. Повышение эффективности ТЭСОТ возможно как за счет получения новых термоэлектрических материалов с улучшенными показателями добротности [2-4], так и путем оптимизации конструкции и режимов работы [5,6]. Конструктивно ТЭСОТ состоит из ТЭМ, теплораспределителя, обеспечивающего теплопередачу от ТНЭ к холодной стороне ТЭМ, и устройства (кулера) отвода теплоты от горячей стороны ТЭМ в окружающее пространство (рис. 1). Эффективность ТЭСОТ зависит от рабочих характеристик и параметров всех элементов конструкции и их взаимного влияния. Режимы термоэлектрического охлаждения в зависимости от рабочих характеристик ТЭМ и термического сопротивления теплоотводящей системы были исследованы в работах [5,6]. В [5] также было показано, что термическое сопротивление теплораспределителя оказывает существенное влияние на температуру ТНЭ. Целью настоящей работы является численное моделирование теплообмена в объеме теплораспределителя, расчет его термического сопротивления и оптимизация параметров.

\section{Постановка задачи}

Идеальным вариантом теплопередачи от ТНЭ к ТЭМ является конфигурация, когда имеется полное соответствие их габаритов, в этом случае ТНЭ может крепиться непосредственно на холодную сторону ТЭМ. Однако для эффективного охлаждения мощных и миниатюрных ТНЭ требуются, как правило, ТЭМ бо́льшего размера, поскольку холодопроизводительность ТЭМ имеет прямо пропорциональную зависимость от суммарной площади сечения ветвей. Из-за несоответствия габаритов ТЭМ и посадочной площадки ТНЭ возникает неоднородность полей температуры и теплового потока, и это негативным образом влияет на эффективность охлаждения и тепловой режим ТНЭ. Для уменьшения данного эффекта между ТНЭ и ТЭМ размещается теплораспределитель, в качестве которого обычно используется прямоугольная теплораспределяющая пластина (ТРП) из материала с высоким значением коэффициента теплопроводности. Такие ТРП изготавливаются как из металлов (медь, алю-

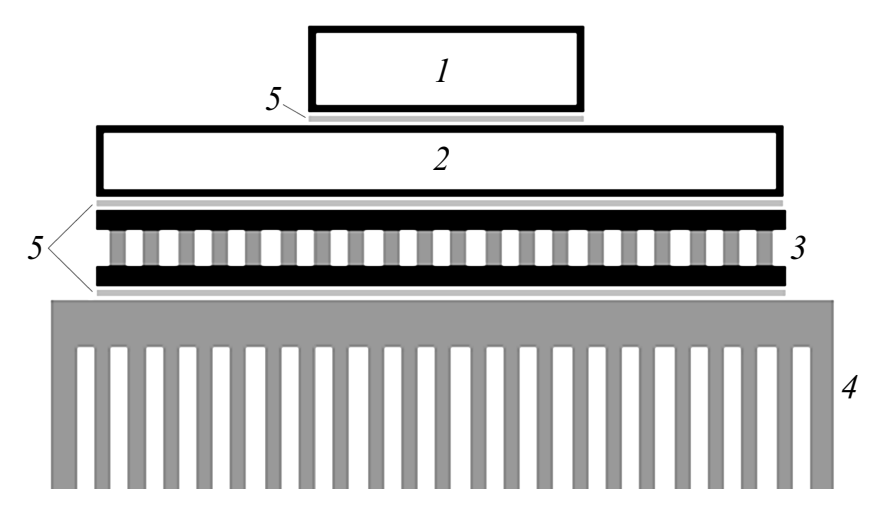

Рис. 1. Схема термоэлектрической системы охлаждения и терморегулирования: 1 - ТНЭ, 2 - теплораспределитель, 3 - ТЭМ, 4 - кулер, 5 - тепловые контакты. 
миний), так и диэлектрических материалов [7]. В качестве теплораспределителей могут быть также использованы устройства (гипертеплопроводящие панели, плоские тепловые трубы), в которых высокая эффективность теплопередачи в поперечных направлениях обеспечивается за счет движения теплоносителя в пористой среде и его фазовых превращений $[8,9]$.

Определим неоднородность температуры в объеме ТРП и степень ее влияния на эффективность охлаждения при различных значениях коэффициента теплопроводности материала $\lambda$ и толщины ТРП. Расчеты температурного поля в объеме ТРП проведены на основе численного решения уравнения теплопроводности вида

$$
c \rho \frac{\partial T}{\partial t}=\lambda\left(\frac{\partial^{2} T}{\partial x^{2}}+\frac{\partial^{2} T}{\partial y^{2}}+\frac{\partial^{2} T}{\partial z^{2}}\right),
$$

где $c, \rho-$ удельная теплоемкость и плотность материала, $T$ - температура, $t-$ время, $x, y, z-$ пространственные координаты. Уравнение (1) дополнено граничными условиями второго рода

$$
\left.\lambda \frac{\partial T}{\partial l}\right|_{l=0, L}=\left.q\right|_{l=0, L}
$$

где $l=x, y, z, L-$ размер ТРП, соответствующий этим координатам. На верхней стороне ТРП по месту контакта с ТНЭ задается однородный тепловой поток $q$, величина которого определяется отношением тепловой мощности, поступающей от ТНЭ, к его посадочной площади. С нижней стороны распределение теплового потока, отводимого от ТРП, рассчитывается исходя из холодопроизводительности ТЭМ с учетом его нагрузочной характеристики $Q\left(\Delta T_{\mathrm{TEM}}\right)$. Теплообмен боковых сторон и части свободной верхней поверхности ТРП с внешней средой не учитывался.

Для решения уравнений (1) и (2) применялся метод суммарной аппроксимации с расщеплением задачи по пространственным координатам $[10,11]$. Из решения (1) и (2) определялось установившееся стационарное распределение температуры в объеме ТРП.

При проведении расчетов рассматривался серийный ТЭМ „РЕ-287-10-15“, имеющий поперечные габариты $40 \times 40 \mathrm{~mm}^{2}$. Его холодопроизводительность задавалась с помощью нагрузочной прямой $Q\left(\Delta T_{\mathrm{TEM}}\right)[5,6]$, соответствующей силе тока $I=2.7 \mathrm{~A}$ и имеющей граничные значения $Q=69 \mathrm{~W}$ при температурном перепаде $\quad \Delta T_{\mathrm{TEM}}=0 \quad$ и $Q=0 \quad$ при $\quad \Delta T_{\mathrm{TEM}}=72.5^{\circ} \mathrm{C}$. В ТЭМ поперечное сечение ветвей мало $\left(1 \mathrm{~mm}^{2}\right.$ у „PE-287-10-15“6) по сравнению с его общей площадью $\left(1600 \mathrm{~mm}^{2}\right)$, что позволяет считать распределения холодопроизводительности и температур на сторонах ТЭМ непрерывными функциями от координат $x$ и $y$. При этом предполагается локальное равенство холодопроизводительности ТЭМ и нормальной составляющей теплового потока на поверхности ТРП в каждой точке поверхности контакта. Температура горячей стороны ТЭМ в общем случае определяется характеристиками теплоотводящей системы и окружающей среды, в данных расчетах она полагалась равной $25^{\circ} \mathrm{C}$.

Исследование процесса теплопередачи проведено для случая, когда поперечные габариты ТЭМ превышают размеры ТНЭ. Расчеты проведены при следующих параметрах: поперечных размерах ТРП по осям $x$ и $y$ $40 \times 40 \mathrm{~mm}^{2}$, разных толщинах $\delta($ ось $z)$, кратных величинах коэффициента теплопроводности материала $\lambda=200$ и $400 \mathrm{~W} /(\mathrm{m} \cdot \mathrm{K})$, близких к значениям алюминия и меди. ТНЭ, имеющий мощность тепловыделения $45 \mathrm{~W}$ и квадратную посадочную площадку со стороной $a$, расположен в центре ТРП. С учетом симметрии задачи относительно осей $x$ и $y$ в качестве расчетной области рассмотрена $1 / 4$ часть объема пластины.

\section{Обсуждение результатов}

Результаты расчета температурного поля при $\delta=2 \mathrm{~mm}, \lambda=200 \mathrm{~W} /(\mathrm{m} \cdot \mathrm{K})$ и $a=10 \mathrm{~mm}$ приведены на рис. 2. На рисунке представлены двумерные температурные распределения в среднем сечении плоскости $x z \quad(a)$ и верхней поверхности ТРП в
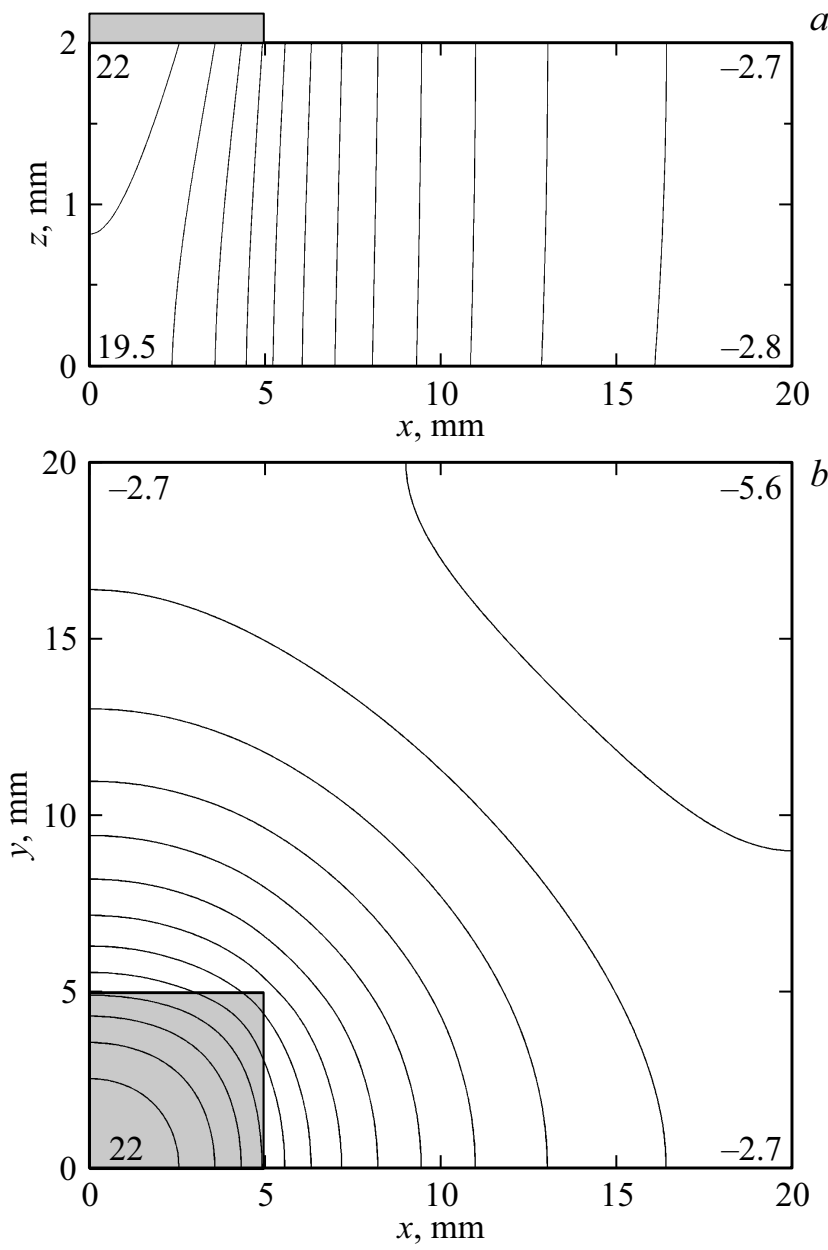

Рис. 2. Распределения $T(x, z)$ в среднем сечении ТРП $(a)$ и $T(x, y)$ на верхней стороне ТРП $(b)$. 
плоскости $x y(b)$, шаг между изолиниями равен $2^{\circ} \mathrm{C}$, также указаны значения температуры, соответствующие угловым точкам. Серой областью на верхней границе обозначено месторасположение ТНЭ, нижняя сторона контактирует с холодной стороной ТЭМ (рис. 2,a). Температурное поле в ТРП является существенно неоднородным, максимальное значение температуры на верхней стороне находится в центре под элементом и равно $T_{\max }=22^{\circ} \mathrm{C}$, минимальное значение - в наиболее удаленной, угловой точке $T_{\min }=-5.6^{\circ} \mathrm{C}$ (рис. $2, b$ ). Перепад температуры по толщине ТРП $\Delta T_{z}$ обусловлен термическим сопротивлением слоя материала, величина перепада переменна по площади (под элементом $\Delta T_{z}=2.5^{\circ} \mathrm{C} \quad$ и на краях $\left.\Delta T_{z}=0.1^{\circ} \mathrm{C}\right)$, поскольку зависит от распределения плотности теплового потока.

Неоднородность температуры в ТРП в значительной степени зависит от поперечного размера ТНЭ. На рис. 3 приведены зависимости от $a$ минимальных (кривые 1 и 2) и максимальных (кривые 3 и 4) значений температуры на нижней (пунктирные линии) и верхней (сплошные линии) поверхностях ТРП для приведенных выше параметров. Как видно из рисунка, максимальные значения температуры по сравнению с минимальными имеют значительно более сильную зависимость от поперечного размера а посадочного места ТНЭ. Это связано с тем, что основная доля тепловой мощности передается по площади и ближайшей окрестности посадочного места ТНЭ, на периферии ТРП плотность теплового потока существенно ниже. Предельному случаю $a=40 \mathrm{~mm}$ соответствует равенство размеров ТЭМ и ТНЭ, при котором распределение теплового потока и температуры в поперечном сечении ТРП становится однородным, при этом по толщине ТРП температурный перепад, обусловленный термическим сопротивлением слоя материала, составляет $\Delta T_{z}=0.3^{\circ} \mathrm{C}$.

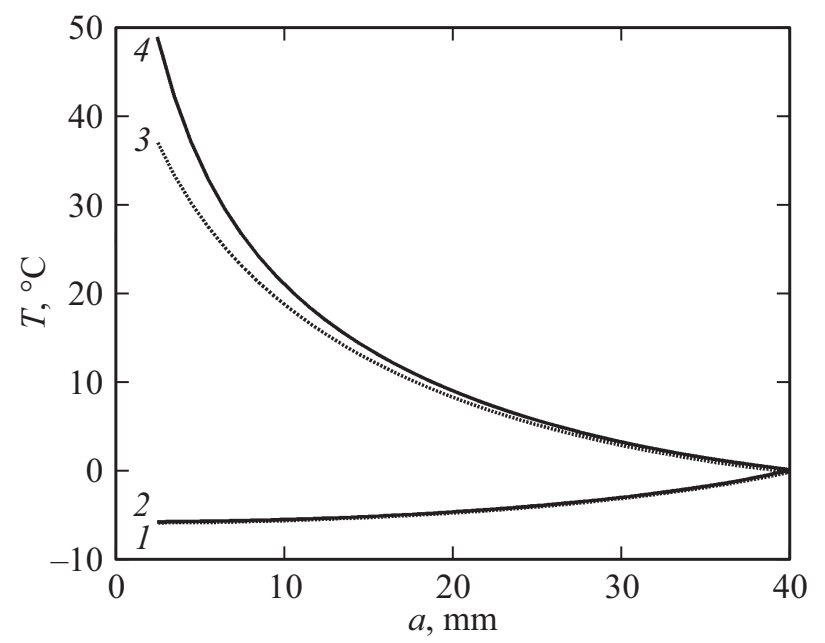

Рис. 3. Зависимости минимальных (кривые 1 и 2) и максимальных (кривые 3 и 4) значений температуры на нижней (пунктирные линии) и верхней (сплошные линии) поверхностях ТРП.

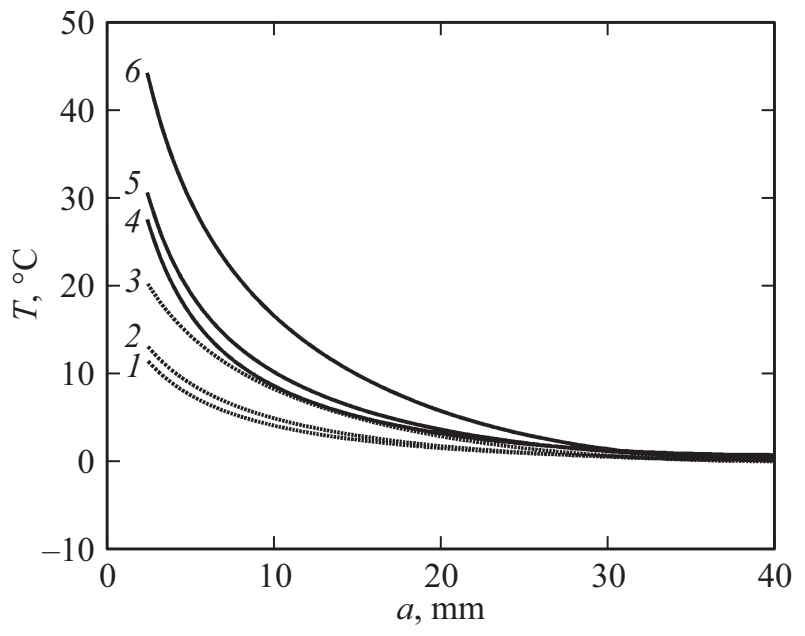

Рис. 4. Зависимости средней температуры по посадочному месту ТНЭ: кривые 1,2 и 3 соответствуют $\delta=6,4$ и $2 \mathrm{~mm}$ при $\lambda=400 \mathrm{~W} /(\mathrm{m} \cdot \mathrm{K})$, кривые 4,5 и 6 соответствуют $\lambda=200 \mathrm{~W} /(\mathrm{m} \cdot \mathrm{K})$ и тем же $\delta$.

Теплопередача на границах ТНЭ-ТРП и ТРП-ТЭМ происходит по всей площади контактных поверхностей, поэтому характеристики теплообмена в ТРП в большей степени определяются не значениями локальных максимумов и минимумов температуры, а средними значениями. На рис. 4 показаны зависимости средней температуры по посадочному месту ТНЭ от величины $a$ : кривые 1-3 получены для значений $\delta=6,4$ и $2 \mathrm{~mm}$ при теплопроводности меди $\lambda=400 \mathrm{~W} /(\mathrm{m} \cdot \mathrm{K})$, кривые 4-6 соответствуют теплопроводности алюминия $\lambda=200 \mathrm{~W} /(\mathrm{m} \cdot \mathrm{K})$ и тем же значениям $\delta$. Средняя температура нижней поверхности ТРП, контактирующей с ТЭМ, для всех $\delta$ и $\lambda$ не зависит от $a$ и примерно равна $-0.2^{\circ} \mathrm{C}$. Постоянство средней температуры нижней поверхности обусловлено линейным видом нагрузочной характеристики и однородной температурой теплотвода, при этом центральная область ТЭМ, имеющая более высокую холодопроизводительность при меньшем температурном перепаде, полностью компенсирует пониженную производительность периферийных участков, работающих при увеличенном температурном перепаде.

Интегральным параметром, характеризующим теплопередающую способность теплораспределителя, является его термическое сопротивление $R_{T}$. Количественно термическое сопротивление рассчитывается как отношение разности средних температур посадочного места ТНЭ и нижней поверхности ТРП к передаваемой тепловой мощности $Q$. При этом величина $R_{T}$ зависит как от теплопроводности материала ТРП, так и размеров ТНЭ и ТРП. На рис. 5 приведены зависимости $R_{T}$ от габарита посадочного места ТНЭ $а$ при значениях коэффициента теплопроводности $\lambda=200$ (сплошные линии), $400 \mathrm{~W} /(\mathrm{m} \cdot \mathrm{K})$ (пунктирные линии) и толщины $\delta=6$ (кривые 1 и 4), 4 (кривые 2 и 5) и $2 \mathrm{~mm}$ (кривые 3 


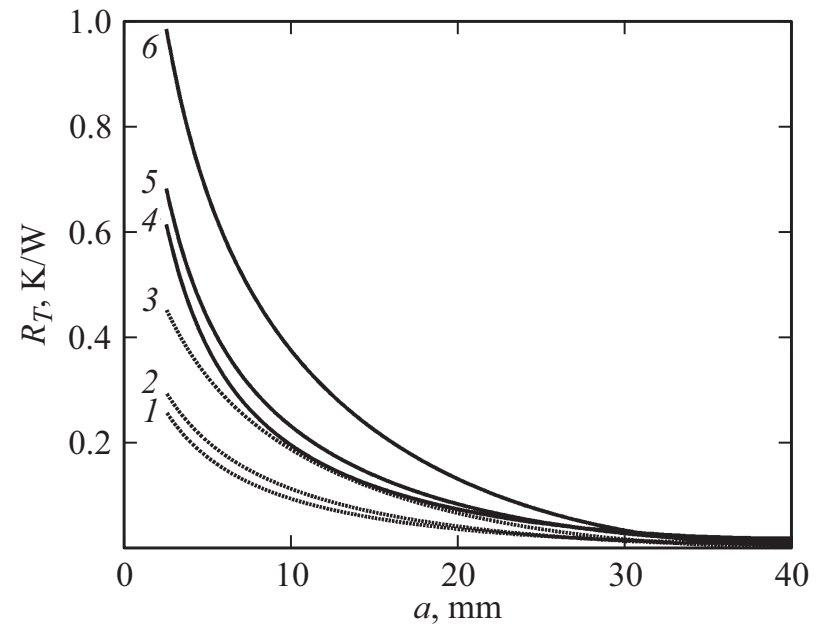

Рис. 5. Зависимости $R_{T}$ (a) при $\lambda=200$ (сплошные линии), $400 \mathrm{~W} /(\mathrm{m} \cdot \mathrm{K})$ (пунктирные линии) и толщине $\delta=6$ (кривые 1 и 4 ), 4 (кривые 2 и 5 ) и $2 \mathrm{~mm}$ (кривые 3 и 6 ).

и 6). Для всех случаев величина $R_{T}$ монотонно падает при увеличении $a$.

В целом величина $R_{T}$ определяется распределениями температуры и плотности теплового потока, сформировавшимися в зависимости от параметров ТРП и ТНЭ. В величине $R_{T}$ можно условно выделить две составляющие, одна из которых $R_{T z}$ связана с теплопередачей по оси $z$ в объеме виртуального параллелепипеда, верхняя грань которого является посадочным местом ТНЭ, другая $R_{T x y}$ с растеканием теплоты в периферийные области ТРП через боковые грани этого параллелепипеда в направлениях $x$ и $y$. Оценим влияние этих составляющих на основе сравнения двух вариантов ТРП с $\delta=4 \mathrm{~mm}, \lambda=200 \mathrm{~W} /(\mathrm{m} \cdot \mathrm{K})$ и $\delta=2 \mathrm{~mm}, \lambda=400 \mathrm{~W} /(\mathrm{m} \cdot \mathrm{K})$. Составляющая $R_{T x y}$ обратно пропорциональна произведению площади боковых граней параллелепипеда и коэффициента теплопроводности $4 a \delta \lambda$, относительно величины $R_{T x y}$ оба рассматриваемых варианта ТРП равнозначны. Составляющая $R_{T z}$ пропорциональна $\delta / a^{2} \lambda$, соответственно ее величина для алюминиевой ТРП в 4 раза больше, чем для медной. Следовательно, именно вклад составляющей $R_{T z}$ обусловливает различие в величине полного термического сопротивления для этих вариантов ТРП. Сопоставление кривых 3 и 5 (рис. 5) показывает наибольшую разницу в значениях $R_{T}$ при малых $a$, при увеличении $a$ зависимости сближаются. Так, при $a=2.5 \mathrm{~mm}$ значение $R_{T}$ для алюминиевой ТРП на $50 \%$ выше, чем для медной, при $a=20 \mathrm{~mm}$ величина такого превышения примерно в 2 раза меньше. При этом медная ТРП во всем диапазоне $a$ имеет меньшие значения $R_{T}$, поэтому является более эффективным теплораспределителем, чем алюминиевая ТРП удвоенной толщины при прочих равных параметрах.

Зависимости $R_{T}$ от толщины ТРП $\delta$ показаны на рис. 6 (алюминий) и 7 (медь) для различных значений $a$, кривые $1-5$ соответствуют значениям $22.5,17.5,12.5,7.5$ и $2.5 \mathrm{~mm}$. Пунктирной линией на рисунках приведена зависимость для случая однородного теплового потока, соответствующего значению $a=40 \mathrm{~mm}$. Величина $R_{T}$ для медной ТРП, как и следовало ожидать, вдвое ниже, чем для алюминиевой. Зависимости $R_{T}(\delta)$, соответствующие фиксированным $a$, имеют минимумы, которые обусловлены наличием двух разнонаправленных, конкурирующих тенденций для рассмотренных выше составляющих $R_{T x y} \sim \delta^{-1}$ и $R_{T z} \sim \delta$. Увеличение $\delta$, с одной стороны, способствует более эффективному растеканию теплоты и снижению значений $R_{T x y}$ и общего термического сопротивления $R_{T}$, с другой стороны, оно приводит к пропорциональному росту как $R_{T z}$, так и $R_{T}$ в целом. Минимумы зависимостей на рис. 6 и 7 имеют следующие координаты: для алюминия 6.3, 7.8, 9 и $10.3 \mathrm{~mm}$, для меди $6.4,7.9,9.1$ и $10.3 \mathrm{~mm}$ соответственно для кривых 1-4. Кривые 5 в рассматриваемом диапазоне $\delta$

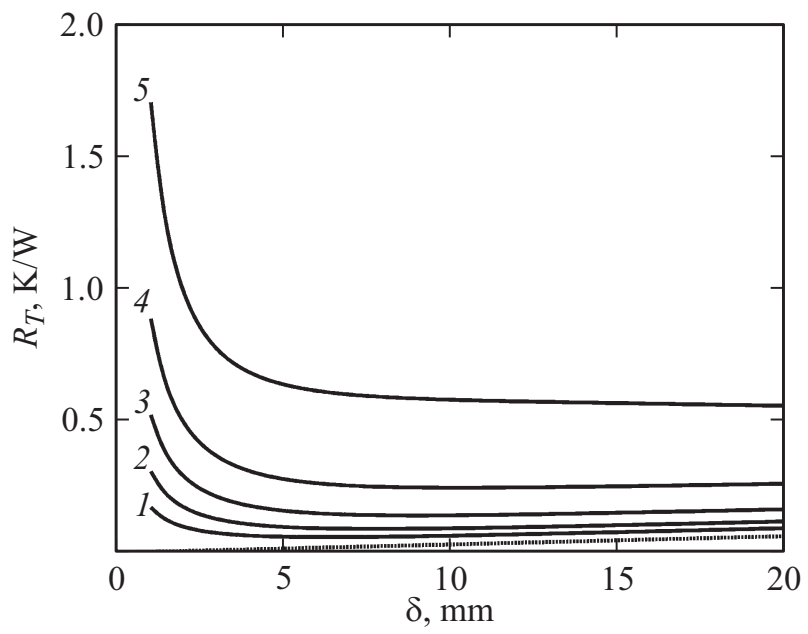

Рис. 6. Зависимости $R_{T}(\delta)$ для алюминия, кривые 1-5 соответствуют $a=22.5,17.5,12.5,7.5$ и $2.5 \mathrm{~mm}$.

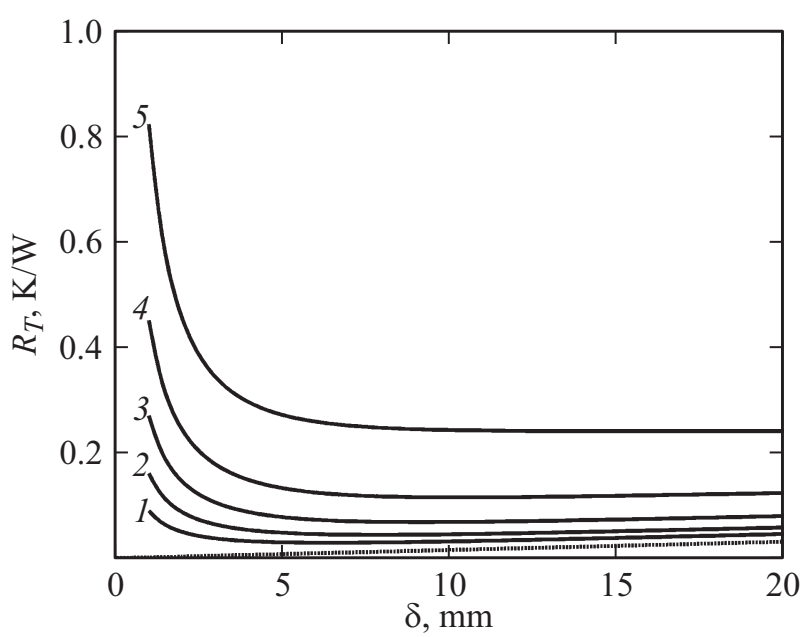

Рис. 7. Зависимости $R_{T}(\delta)$ для меди, кривые $1-5$ соответствуют $a=22.5,17.5,12.5,7.5$ и $2.5 \mathrm{~mm}$. 
минимумов не имеют. Вблизи минимумов зависимости имеют пологий характер, поэтому при определении параметров конструкции теплораспределителя предпочтительнее выбирать значения $\delta$ на левых падающих ветвях зависимостей, где значения $R_{T}$ близки к минимальным, а толщина и масса ТРП заметно ниже. С учетом данных соображений диапазон оптимальных значений $\delta$ составляет примерно от $3 \mathrm{~mm}$ при $a=22.5 \mathrm{~mm}$ до $5 \mathrm{~mm}$ при $a=2.5 \mathrm{~mm}$. Следует отметить, что на выбор материала и геометрических параметров теплораспределителя также влияют конструкционные, технологические и экономические требования, а также „жесткость“ условий по температурному режиму ТНЭ.

\section{Заключение}

Проведено численное моделирование теплообмена в теплораспределителе ТЭСОТ. Получены распределения температуры с учетом мощности тепловыделения и размера ТНЭ, параметров ТРП, нагрузочных характеристик ТЭМ. Расчет температурного сопротивления ТРП проведен по средним температурам посадочного места ТНЭ и нижней поверхности ТРП. Результаты расчетов показали, что медная ТРП во всем диапазоне $a$ имеет меньшие значения $R_{T}$ и следовательно является более эффективным теплораспределителем, чем алюминиевая ТРП удвоенной толщины. Установлено, что в зависимости от размера ТНЭ оптимальная толщина ТРП как для меди, так и алюминия составляет 3-5 mm. Оптимизация параметров теплораспределителя позволяет минимизировать температурный перепад и тем самым снизить температуру ТНЭ. Результаты работы, полученные применительно к ТЭСОТ, также могут быть использованы для обычной системы охлаждения, имеющей теплораспределитель подобной конструкции.

Результаты исследований, представленные в настоящей и ранее опубликованных $[5,6]$ работах, позволяют проводить расчет и оптимизацию режимов ТЭСОТ с учетом мощности тепловыделения ТНЭ, рабочих характеристик ТЭМ, параметров теплораспределителя и кулера с целью достижения максимальной эффективности охлаждения и снижения энергозатрат.

Исследование выполнено при финансовой поддержке Российского фонда фундаментальных исследований, Правительства Красноярского края, Красноярского краевого фонда поддержки научной и научно-технической деятельности в рамках научного проекта № 16-41-242104.

\section{Список литературы}

[1] Ненашев А.П. Конструирование радиоэлектронных средств. М.: Высшая школа, 1990. 432 с.

[2] Булат Л.П., Новотельнова А.В., Тукмакова А.С., Ережеп Д.Е., Освенский В.Б., Сорокин А.И., ПшенайСеверин Д.А., Аимонтас С. // ЖТФ. 2017. Т. 87. Вып. 4. C. 584-592.
[3] Дмитриев А.В., Звягин И.П. // УФН. 2010. Т. 180. № 8. C. $821-838$.

[4] Патрушева Т.Н., Подорожняк С.А., Шелованова Г.Н. // Журнал Сибирского фед. ун-та. Сер. „Техника и технология“". 2013. Т. 6. № 6. С. 657-664.

[5] Васильев Е.Н. // ЖТФ. 2017. Т. 87. Вып. 1. С. 80-86.

[6] Васильев Е.Н. // ЖТФ. 2017. Т. 87. Вып. 9. С. 1290-1296.

[7] Глинский И.А., Зенченко Н.В. // Микроэлектроника. 2015. T. 44. № 4. C. 269-274.

[8] Васильев Е.Н., Никифорова Е.С. // Вестник Сибир. гос. аэрокосм. ун-та. 2005. Вып. 3. С. 23-26.

[9] Васильев Е.Н., Деревянко В.А., Нестеров Д.А., Косенко B.E., Чеботарев В.E. // Вычислительные технологии. 2009. T. 14. № 6. С. 19-28.

[10] Самарский А.А. Теория разностных схем. М.: Наука, 1989. $656 \mathrm{c.}$

[11] Дульнев Г.Н., Парбенов В.Г., Сигалов А.В. Применение ЭВМ для решения задач теплообмена. М.: Высшая школа, 1990. $207 \mathrm{c}$. 